\title{
Aontia: un antico toponimo dalle Mappe Aragonesi
}

\author{
Aontia: an ancient toponym from the Aragon maps
}

\begin{abstract}
Antonio Pecci ${ }^{a}$, Ida Campanile ${ }^{b}$
${ }^{\text {a }}$ Dipartimento di Scienze Umane - Università degli studi della Basilicata, Potenza, Italy, antonio.pecci@unibas.it

b Dipartimento di Culture Europee del Mediteraneo - Università degli studi della Basilicata, Matera, Italy, ida.campanile@unibas.it
\end{abstract}

\begin{abstract}
The Aragon geographical maps represent the territory of the ancient Kingdom of Naples. they date back to the second half of the fifteenth century, probably some of them or some copies were subsequently modified or updated. These ancient maps were rediscovered about thirty years ago in the State Archives of Naples and in the Bibliothèque Nationale de France in Paris, and they have been under study for some years. They are unfortunately still little used in the scientific field, although several contributions have demonstrated their validity as an investigation tool thanks to their undoubted information potential. In fact, thanks to the very high degree of characterization of these maps it is possible to advance hypotheses and considerations of a historical-archaeological nature of the territories they represent. It is often toponymic analysis that offers insights and guides the early stages of research: toponyms relating to natural and anthropic elements inform about landscapes rich of medieval and classical references. The case study proposed here relates to the toponym Aontia, located on the Aragon maps near the centers of the Basilicata of Cirigliano and Gorgoglione. It is a place currently unidentified and not attested in any medieval or modern source; its toponym may refer to some references relating to an epithet of the wellknown Greek divinity Artemis and to the presence of a sanctuary dedicated to it or to an ancient settlement. Starting from the analysis of the toponym Aontia, a localization proposal will be carried out based on the etymological and historical study, on the topographic survey and on the remote sensing analysis.
\end{abstract}

Keywords: Historical Cartography, classical and roman archeology, Aragon maps, Basilicata.

\section{Introduzione}

Le Mappe Aragonesi (Valerio, Greca, 2008; Vitolo, 2016a) raffigurano il territorio dell'antico Regno di Napoli, e sono generalmente datate alla seconda metà del 1500; non è escluso, tuttavia, che su di esse siano state effettuate delle successive modifiche o aggiornamenti (Valerio, La Greca, 2008; Valerio, 2015, 2016; Vitolo, 2016b; Ambrosio 2016; Pecci, 2018). Diversamente dalle contemporanee mappe della fine del Quattrocento, contraddistinte dall'elevato numero di errori e da considerevoli deformazioni, le
Mappe Aragonesi presentano una maggiore precisione e sono ricche di dettagli corografici. Queste antiche e preziose carte furono riscoperte da Vladimirio Valerio circa tre decenni fa nella Bibliothèque Nationale de France a Parigi (BNF) e nell'Archivio di Stato di Napoli (ASN) e, oramai da diversi anni, sono al centro di dibattiti e ricerche. Attualmente, si citano diversi contributi scientifici sull'utilizzo delle stesse anche per finalità archeologiche (Greca, 2008, 2016; Pellicano, 2010; Capano, 2011, 2012; Scarpa, 
2014; Castellano 2016; Gargano, 2016; Pecci, 2018) che hanno dimostrato l'enorme potenziale in tale ambito di ricerca. Queste mappe si rivelano, infatti, molto preziose per l'elevato numero di toponimi, relativi ad elementi antropici e naturali, che sanno "raccontare" un paesaggio in cui si fondono e sovrappongono riferimenti antichi o medievali.

Il caso di studio qui proposto fa riferimento al toponimo Aontia (BNF, Cartes et Plans, GE AA 1305-4), situato sulle Mappe Aragonesi vicino ai centri della Basilicata di Cirigliano e di Gorgoglione. È un luogo attualmente non identificato e non attestato in alcuna fonte medievale o moderna; il suo toponimo potrebbe essere connesso ad alcuni riferimenti relativi ad un epiteto della ben nota divinità greca Artemide e alla presenza di un santuario ad esso dedicato o di un antico insediamento. A partire dall'analisi del toponimo Aontia, verrà effettuata, attraverso un approccio scientifico interdisciplinare, una proposta di localizzazione basata sullo studio etimologico e storico, sull'indagine topografica e sull'analisi da telerilevamento.

\section{Aontia sulle Mappe Aragonesi}

Il toponimo Aontia è scritto in nero sulle Mappe Aragonesi. Il primo carattere, dagli scriventi interpretato come una "A", appare però alquanto anomalo in quanto risulta border line tra le "A" e le "O", ed è diverso da altre lettere scritte in maiuscolo e dislocate all'interno della Mappa. Anche la seconda lettera non è di facile lettura ma, in base ad una attenta analisi calligrafica, è identificabile come una "o". Al di sotto del toponimo sono state disegnate cinque piccole casette dipinte in rosso, il che significherebbe, in base alla lettura canonica delle antiche carte, la presenza di un piccolissimo abitato, il quale risulta tra i più piccoli raffigurati in tutta la regione. Questo minuscolo nucleo insediativo si colloca su un'altura, a poca distanza da una sorgente, e sulla sinistra di un affluente del Rivo di Cirilliano, un corso d'acqua che nasce ad est di Castelmediano (l'attuale Castelmezzano), che è a sua volta tributario del ben più importante Fiume Acri (il fiume Agri). I centri abitati più vicini ad Aontia, attualmente conosciuti, sono
Gorgollione (Gorgoglione), a sud, Cirilliano (Cirigliano), a sud-est, la Accettura (Accettura), a nord; rimane sconosciuta l'ubicazione di Appisio, a sud-ovest.

\section{Identificazione di Aontia da remote sensing}

Le Mappe Aragonesi sono caratterizzate da una prospettiva a volo di uccello: in tale raffigurazione il suolo viene riprodotto come se fosse osservato con un angolo visuale di $45^{\circ}$, genericamente rivolto verso sud. Questa rappresentazione prospettica, unita ad una deformazione e imprecisione geografica delle Mappe, rende quasi impossibile la sovrapposizione delle stesse sulla contemporanea cartografia. Di conseguenza, per una ben più facile lettura e interpretazione, è stato opportuno utilizzare un software come Google Earth, il quale permette una visualizzazione tridimensionale del paesaggio e di osservare il terreno attraverso una prospettiva a volo di uccello. L'areale in cui si colloca Aontia sulle Mappe è stato messo a confronto con Google Earth e, partendo dal riconoscimento di alcuni punti corografici (Garaguso, Cirigliano, fiumare, alture, etc.), è stato possibile riconoscere su Earth, sommariamente, una porzione di paesaggio visibilmente simile a quella riprodotta sulle antiche mappe (Fig. 1).

Le coordinate geografiche della presunta zona di ubicazione dell'antico toponimo sono state riportate all'interno di un GIS, in cui sono stati consultati e confrontati tra loro diversi layer relativi a foto aeree storiche, immagini satellitari, carte topografiche, DTM (Digital Surface Model) e DSM (Digitale Surface Model). Partendo dalla lettura della cartografia dell'Istituto Geografico Militare, precisamente dalle tavolette IGM serie 25V del 1956 (200 III-NE ACCETTURA; 200 III-SE GORGOGLIONE), è stato possibile identificare l'area individuata, nella quale ricadrebbe Aontia.

Si tratterebbe dell'odierna "Tempa dei Greci" (Fig. 2), un'altura a circa 1000 m.s.l.m., estesa su circa 70 ettari, con un toponimo dal forte richiamo archeologico, nonostante non vi siano mai stati rintracciati, a quanto risulta, rinvenimenti archeologici (Carucci, 2011). 


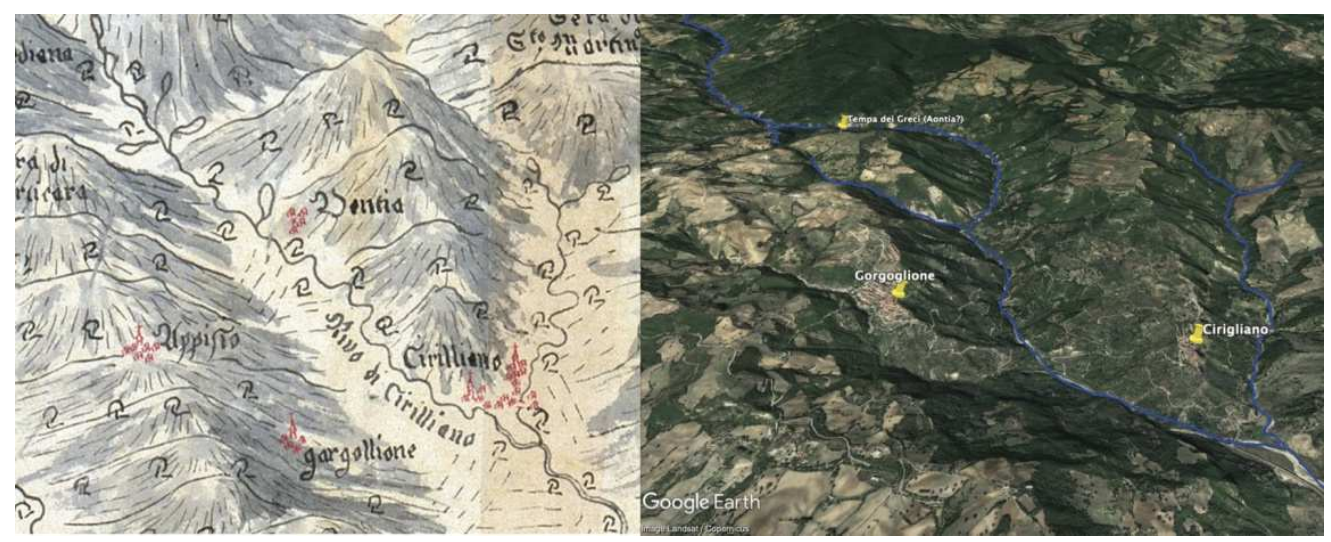

Fig. 1. Confronto tra le Mappe Aragonesi e Google Earth.

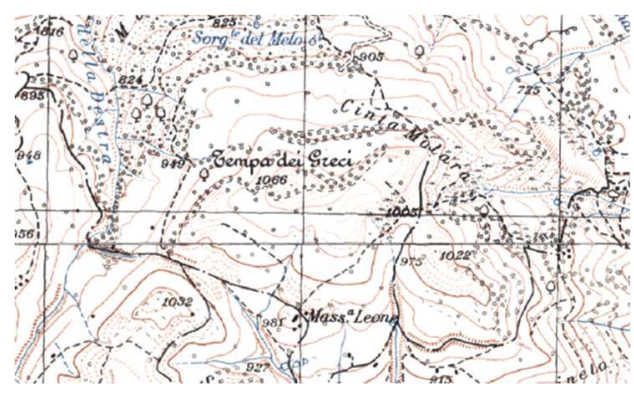

Fig. 2. Tempa dei Greci. IGM serie 25V del 1956 (200 III-NE ACCETTURA; 200 III-SE GORGOGLIONE).

Attualmente è compresa nel territorio amministrativo di Garaguso (Mt); ubicata a nord dello stesso comune lucano, è contraddistinta da un poggio centrale (1066 m.s.l.m.) sopraelevato rispetto a dei plateau dolcemente digradanti (Fig. 3). Dall'osservazione delle varie mappe IGM si riscontra come la zona sia servita da antichi tratturi e sentieri, da una sorgente ("Sorg.te del Melo") probabilmente segnalata dalle stesse Carte Aragonesi, e come l'intera altura sia interamente circondata da rivoli d'acqua confluenti nella "Fiumara di Garaguso" (Rivo di Cirigliano sulle Carte).

Di particolare interesse è un altro toponimo, collocato subito a nord-est dell'altura, "Cinta Molara", che suggerisce la presenza di una cinta muraria il cui ricordo sarebbe rimasto agganciato al toponimo del luogo. In relazione a questa osservazione, appare alquanto interessante la presenza di alcune anomalie, leggibili nel DTM. Queste ipotetiche feature archeologiche, per via della loro forma e dimensione, porterebbero a suppore la presenza di una cinta muraria (se non di più una!) che corre lungo l'altura di Tempa dei Greci (Figg. 3 e 4 ).

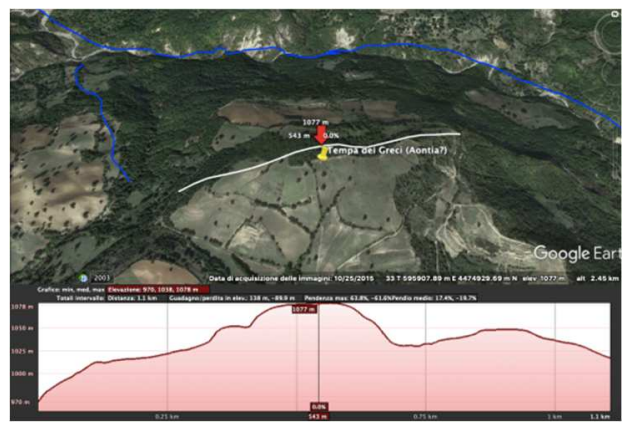

Fig. 3. DTM di Tempa dei Greci. Precisione $1 \mathrm{~m} /$ pixel. Risorse online da http://rsdi.regione.basilicata.it



Fig. 4. Tempa dei Greci. Foto aerea anno 1994.

A giustificare ancor più questa ipotesi, è la presenza di alcuni blocchi lapidei pseudo isodomi ubicati a S dell'area (Fig. 4), lungo una delle 
anomalie rilevate, che per forma e dimensione sarebbero riconducibili ad epoca lucana (V-II secolo a.C.), e confrontabili con le note cinte di Monte Torretta di Pietragalla (Pietragalla, PZ), Monte Croccia (Oliveto Lucano, Mt) e Serra di Vaglio (Vaglio di Basilicata, Pz).

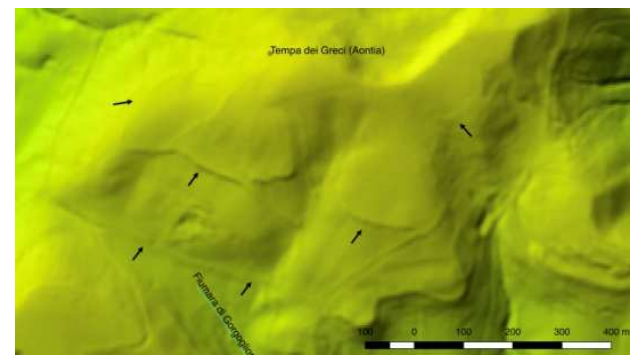

Fig. 5. DTM di Tempa dei Greci. Precisione $1 \mathrm{~m} /$ pixel. Risorse online da http://rsdi.regione.basilicata.it

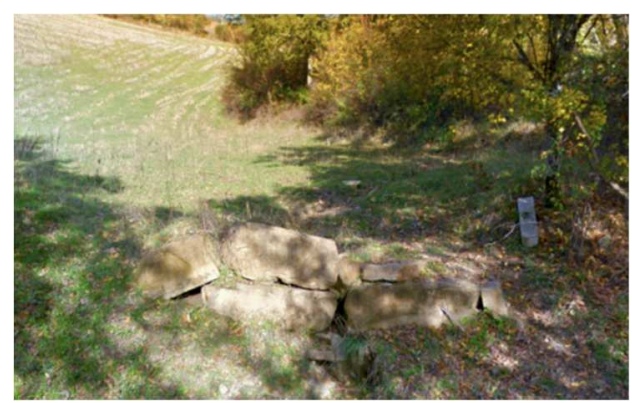

Fig. 6. Blocchi lapidei di forma pseudo isodoma in situ.

\section{L'indagine linguistica e il contesto storico- archeologico}

Il toponimo Aontia presente sulle mappe aragonesi, e di cui si propone l'identificazione con il sito "Tempa dei Greci", ha immediatamente destato curiosità ed interesse; il termine è stato ricondotto, in questa sede, al greco Aovtía, nel quale, come vedremo, è stato riconosciuto un ipotetico, e poco noto, epiteto della divinità Artemide.

Aovtía è stato per la prima volta associato ad Artemide in occasione di un rinvenimento effettuato presso un santuario in Acaia, che, si pensa, da allora, per l'appunto intitolato ad Artemis Aontia; si tratta di un tempio posto nell'area sacra sul monte Panachaikon, presso Ano Mazaraki, a nord di Rakita, a 1300 m di altitudine, data- to a metà VIII-VII secolo a.C. (Lippolis, Livadiotti, Rocco, 2007).

Risulta necessario, a questo punto, per motivare e sostanziare la natura dell' accostamento del toponimo oggetto di studio all'epiteto, approfondire temi legati all'etimologia di questo attributo connesso alla divinità, la cui unica attestazione è rappresentata, finora, da questo caso.

Come accennato, la divinità tutelare del tempio citato è stata riconosciuta in Artemis Aontia per il rinvenimento di numerosi ex voto, tra cui gioielli e armi da caccia, noto attributo di Artemide (Petropoulos, 1996, 1997, 2002), e, in particolare, durante la campagna del 1996, di uno specchio in bronzo della seconda metà del VI secolo a.C. recante sul manico un'iscrizione riportante il seguente testo:



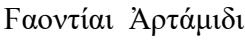

Faovtíar viene, dunque, qui interpretato come

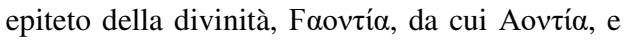
letteralmente tradotto con "che soffia". Si tratta, come accennato, di un epiteto relativo ad Artemide attestato per la prima volta con questa iscrizione, e, da Petropoulos ricondotto alla forma arcaica del participio femminile di ö $\eta \mu$, da tradurre, letteralmente, con "(Artemide) che soffia"; si tratterebbe di un epiteto coniato dai devoti locali in riferimento alla posizione del tempio in una zona costantemente interessata dalla presenza di forti venti, e alla capacità della dea di placarli.

Uno studio di Alonso Dénis del 2009 approfondisce la questione etimologica del presunto epiteto concludendo che qualunque sia l'origine di Faovíál - secondo Alonso Dénis, infatti, l'epiteto Faoviía sarebbe legato ad ö $\mu$, come ha proposto Petropoulos, ma, non come participio femminile; si tratterebbe, piuttosto, di un aggettivo, annoverato come tale grazie a questa iscrizione, derivato dal sostantivo *ö́f $\omega v$, ovvero "vento" - il culto di Artemide "dei venti" avrebbe un parallelo nel Peloponneso. Riporta, infatti, come Pausania (Paus. 4.35.8.), avrebbe avuto la possibilità di visitare a Metone un tempio dedi-

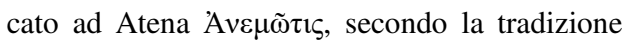


costruito da Diomede per ringraziare la dea che, rispondendo alle sue suppliche, avrebbe calmato i venti che molestavano la regione. In entrambi i

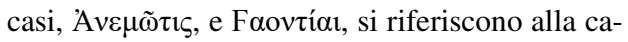
pacità delle dee di placare $i$ venti.

Partendo, quindi, dal presupposto di una riconosciuta pertinenza di Aontia ad Artemide, è ipotizzabile la presenza, in una località riportante tale toponimo, di un luogo (di culto?) riferito a tale divinità che ben rispecchia le caratteristiche pertinenti all'area in cui esso sarebbe stato attestato per la prima volta, trattandosi di un luogo posto, anch'esso, in altura; il sito di Tempa dei Greci è infatti situato a ca 1000 m.s.l.m., particolare che giustificherebbe l'utilizzo, anche in questa sede, di un attributo ("che soffia") della divinità con riferimento ai forti venti riscontrabili in questa zona.

A supporto di questa ipotesi, riguardo l'analisi e l'identificazione del toponimo intercettato sulle mappe aragonesi, subentra un ulteriore elemento.

L'opera di VI secolo d.C. del noto geografo Stefano di Bisanzio, Etnica, riportante alcuni toponimi citati da Ecateo di Mileto, nel segnalare alcune città dell'Enotria interna, fa riferimento ad alcuni siti (Hecat. FGrHist 1 FF 64-71), tra cui, come si riporta di seguito, "Artemision":

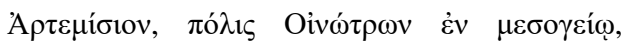



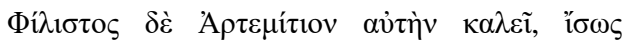

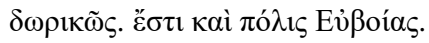

Il toponimo rimanda, anche in questo caso, alla divinità Artemide: letteralmente "Artemisio", "di Artemide", "tempio o luogo consacrato ad Artemide".

Suggestiva è l'ipotesi che il toponimo indicato sulle mappe aragonesi, Aontia, e il toponimo segnalato da Stefano di Bisanzio non ancora geograficamente localizzato, Artemision, entrambi riconducibili ad un luogo legato alla divinità Artemide, possano riferirsi al medesimo sito.

Tali elementi, in virtù di quella che al momento si configura come un'accattivante suggestione, porterebbero ad identificare e, soprattutto, localizzare, la città enotria di Artemision, finora sconosciuta, con l'Aontia riportata nelle carte aragonesi, a sua volta geograficamente collocabile presso "Tempa dei Greci".

Inoltre, se così fosse e, quindi, questa associazione fosse corretta, il toponimo analizzato in questa sede avvalorerebbe ancor più l'ipotesi della natura di questo epiteto che, finora, non trova riscontri altrove, confermando, così, la sua pertinenza alla divinità Artemide.

\section{Conclusioni}

L'analisi delle antiche carte geografiche aragonesi necessita di un approccio olistico che si ponga, quindi, ben oltre la mera lettura corografica. Mediante un approccio interdisciplinare, diacronico e sincronico, lo studio delle Mappe si rivela estremamente proficuo per la ricostruzione del paesaggio storico e per il rinvenimento di antichi centri perduti. L'individuazione del sito archeologico in località Tempa dei Greci, probabilmente Aontia, permette di leggere chiaramente quanto questo ricco patrimonio cartografico continui a comunicarci. La posizione dominante dalla quale era consentita l'osservazione di un ampio areale, la presenza di sorgenti e la collocazione all'interno di un antico sistema viario, si configurano come caratteristiche distintive degli insediamenti lucani; si tratta degli stessi caratteri che contraddistinguono l'altura di Tempa dei Greci. È auspicabile, in futuro, l'avvio di progetti di ricerca sul campo (survey e scavi archeologici) e di indagini non invasive (prospezioni geofisiche) finalizzate alla scoperta di un antichissimo insediamento scomparso, la cui punta dell'iceberg è rappresentata da un tratto di cinta muraria di probabile origine lucana.

\section{Bibliography}

Alonso Déniz, A. (2009). "ARTAMIS ÏAONTIA(SEG XLVIII560)", in Martinez Fernàndez, A., ed., Estudios de Epigrafia Griega, La Laguna, pp. 113-118. 
Ambrosio, A. (2016). "La scrittura delle «Mappe Aragonesi»: riflessioni ed ipotesi”, in Vitolo, G., ed., La rappresentazione dello spazio nel Mezzogiorno aragonese: le carte del Principato Citra, pp. 121-128.

Capano, A. (2011). "La conoscenza e la percezione del territorio nelle carte aragonesi e nella prima cartografia di età moderna: il Potentino", Bollettino Storico della Basilicata, XXVII, pp. 365-404.

Capano, A. (2012). "Conoscenza e percezione del territorio tra cartografia aragonese e prima età moderna: il Materano", Bollettino Storico della Basilicata, XXVIII, pp. 125-158.

Carucci R. (2011). Gorgoglione: l'infanzia, il mito, la storia - Gorgoglione: dal big bang al flysch, Zaccara.

Castellano, M. (2016). "La penisola sorrentina da Castellammare al promontorio di Minerva tra realtà e suggestioni classiche", in Vitolo, G., ed., La rappresentazione dello spazio nel Mezzogiorno aragonese: le carte del Principato Citra, pp. 329-346.

Gargano, G. (2016). "Elementi topografici della costa d'Amalfi”, in Vitolo, G., ed., La rappresentazione dello spazio nel Mezzogiorno aragonese: le carte del Principato Citra, pp. 301-328.

Greca, F. La. (2008). "Antichità classiche e paesaggio medioevale nelle carte geografiche del principato Citra curate da Giovanni Gioviano Pontano. L'eredità della cartografia romana”, in Greca, F. La.; Valerio, V., eds., Paesaggio antico e Medioevale nelle mappe aragonesi di Giovanni Pontano. Le terre del principato Citra, Centro di Promozione Culturale per il Cilento, Acciaroli, pp. 33-78.

Greca, F. La. (2016). “Le mappe aragonesi su pergamena dell’ Archivio di Stato di Napoli e l'eredità cartografica del mondo greco-romano", in Vitolo, G., ed., La rappresentazione dello spazio nel Mezzogiorno aragonese: le carte del Principato Citra, pp. 69-120.

Lippolis, E.; Livadiotti, M.; Rocco, G. (2007). Architettura greca: storia e monumenti del mondo della polis dalle origini al V secolo, p. 649.

Pecci A. (2018). "The Basilicata depicted in the Aragon maps: a gold mine for classical archaeology. An overview of ancient toponymy, roman ruins and natural resources", in Marotta, A.; Spallone, R., eds., FORTMED 2018. PROCEEDINGS of the International Conference on Modern Age Fortification of the Mediterranean Coast, pp. 195-202.

Pellicano, A. (2010). "La Cartografía Aragonesa del Reino de Nápoles en el siglo XV", Revista de estudios colombinos, 6, pp. 43-52.

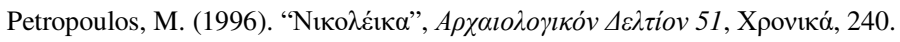

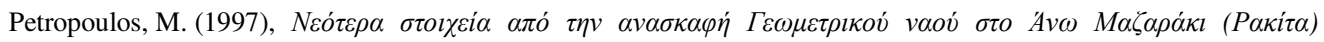

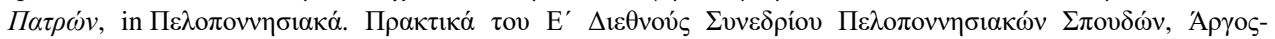

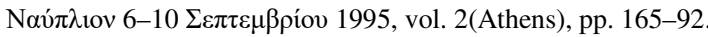

Petropoulos, M., (2002) The geometric temple of Ano Mazaraki (Rakita) in Achaia during the period of

colonisation, in E. Greco (ed.), Gli Achei e l'identità etnica degli Achei d'Occidente, Atti del Convegno Internazionale di Studi (Paestum, 23 - 25 febbraio 2001), Tekmeria III, Paestum - Atene 2002, pp. 143-164

Scarpa, L. (2014). Le due Civitelle nelle mappe aragonesi di Giovanni Pontano, in Annali Storici di Principato Citra XII, 1, pp. 60-79

Valerio, V.; Greca, F. La. (2008). Paesaggio antico e medioevale nelle mappe aragonesi di Giovanni Pontano. Le terre del Principato Citra. Acciaroli: Centro di Promozione Culturale per il Cilento.

Valerio, V. (2015). La cartografia rinascimentale del regno di Napoli: dubbi e certezze sulle pergamene geografiche aragonesi, in Humanistica: an international journal of early Renaissance studies: X, 1/2, pp. 191-232.

Valerio, V. (2016). Le pergamene cartografiche aragonesi del Regno di Napoli: dubbi e certezze, in La rappresentazione dello spazio nel Mezzogiorno aragonese: le carte del Principato Citra (a cura di G. Vitolo), pp. 9-68.

Vitolo, G. (2016a). La rappresentazione dello spazio nel Mezzogiorno aragonese: le carte del Principato Citra, (a cura di) Società Napoletana Di Storia Patria Centro Interuniversitario Per La Storia Delle Città Campane Nel Medioevo, Quaderni, 7.

Vitolo, G. (2016b). Indizi storico-linguistici per la datazione, in La rappresentazione dello spazio nel Mezzogiorno aragonese: le carte del Principato Citra (a cura di G. Vitolo), pp. 129-140. 